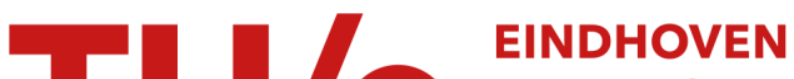 \\ UNIVERSITY OF \\ TECHNOLOGY
}

\section{Recent results on perfect codes and related topics}

Citation for published version (APA):

van Lint, J. H. (1974). Recent results on perfect codes and related topics. In M. Hall Jr., \& J. H. Lint, van (Eds.), Combinatorics, Part 1: theory of designs, finite geometry and coding theory : proceedings of the Advanced Institute on Combinatorics held at Nijenrode Castle, Breukelen, The Netherlands, July 8-20, 1974 (pp. 158-178). (Mathematical Centre Tracts; Vol. 55). Stichting Mathematisch Centrum.

Document status and date:

Published: 01/01/1974

\section{Document Version:}

Publisher's PDF, also known as Version of Record (includes final page, issue and volume numbers)

\section{Please check the document version of this publication:}

-A submitted manuscript is the version of the article upon submission and before peer-review. There can be important differences between the submitted version and the official published version of record. People interested in the research are advised to contact the author for the final version of the publication, or visit the $\mathrm{DOI}$ to the publisher's website.

- The final author version and the galley proof are versions of the publication after peer review.

- The final published version features the final layout of the paper including the volume, issue and page numbers.

Link to publication

\section{General rights}

Copyright and moral rights for the publications made accessible in the public portal are retained by the authors and/or other copyright owners and it is a condition of accessing publications that users recognise and abide by the legal requirements associated with these rights.

- Users may download and print one copy of any publication from the public portal for the purpose of private study or research.

- You may not further distribute the material or use it for any profit-making activity or commercial gain

- You may freely distribute the URL identifying the publication in the public portal.

If the publication is distributed under the terms of Article $25 \mathrm{fa}$ of the Dutch Copyright Act, indicated by the "Taverne" license above, please follow below link for the End User Agreement:

www.tue.nl/taverne

Take down policy

If you believe that this document breaches copyright please contact us at:

openaccess@tue.nl

providing details and we will investigate your claim. 


\title{
RECENT RESULTS ON PERFECT CODES AND RELATED TOPICS
}

\author{
J.H. VAN LINT \\ Technological University, Eindhoven, The Netherlands
}

\section{INTRODUCTION}

In this paper we will use the framework and terminology explained by DELSARTE in the previous paper [3]. We consider perfect codes in the Hamming and Johnson schemes and in association schemes corresponding to distance-transitive graphs. For these schemes we illustrate some recent examples and concentrate on the recent developments concerning nonexistence proofs. Here the main tools are the sphere packing bound (cf. [3, \$ 4.1]) and LIoyd's theorem [3, Theorem 7].

The completely regular codes briefly mentioned by DELSARTE contain two classes which have properties similar to the perfect codes. The most interesting of these properties is that such codes can be used to construct t-designs. The search for such codes started a few years ago. Again we will illustrate some examples and prove some non-existence theorems.

2. HAMMING SCHEMES $H(n, q)$ WITH $q$ A PRIME POWER

Let $q=p^{r}$ (p a prime). We consider a perfect e-exror-correcting code $Y$, i.e. a perfect code of order e. The minimum distance of $Y$ is $d=2 e+1$. For $e=1$ there are many known examples of perfect codes (cf. [7]). For $e>1$ one always has the trivial example $e=n$ and $|y|=1$. For $q=2$ and $\mathrm{n}=2 \mathrm{e}+1$ the repetition code $\mathrm{Y}:=\{(0,0, \ldots, 0),(1,1, \ldots, 1)\}$ provides an example. Besides these there are 2 non-trivial perfect codes known as the Golay codes (cf. [7]). The parameters of these codes are $\mathrm{n}=23, q=2$, 
$e=3$ respectively $n=11 \quad 1=3, e=2$. For both of these codes it was shown recently [4], [12] that they are unique (up to translations and permutations of coordinate places). In 1970 VAN LINT [8] proved that if there are any other perfect codes then they have $e>3$ and $p$ e. A year later TIETÄVÄINEN [13] proved that if $e \geq 3$ and $p \leq e$ then there is no perfect code of order $e$ in $\mathrm{H}(n, q)$, thus completely settling the problem for the case where $q$ is a prime power. The same result was obtained independently by ZINOV'EV \& IEONT'EV [15]. All these theorems had quite complicated proofs. A few months ago TIETÄVÄINEN [14] succeeded in shortening the proof considerably for $q>2$. It turns out that very little more is needed if $q=2$. We shall present the complete proof below.

We remind the reader of the definition of the Krowtchouk polynomial $\mathrm{K}_{\mathrm{k}}(\mathrm{n}, \mathrm{q} ; \mathrm{u})$ of degree $\mathrm{k}$ :

$$
k_{k}(n, q ; u):=\sum_{j=0}^{k}(-1)^{j}(q-1)^{k-j}\left(\begin{array}{l}
u \\
j
\end{array}\right)\left(\begin{array}{l}
n-u \\
k-j
\end{array}\right) \text {. }
$$

The sum polynomial $\Psi_{\mathrm{e}}$ occurring in Lloyd's theorem which we quote below is

$$
\Psi_{e}(x):=k_{e}(n-1, q ; x-1)=\sum_{i=0}^{e}(-1)^{i}\left(\begin{array}{c}
n-x \\
e-i
\end{array}\right)\left(\begin{array}{c}
x-1 \\
i
\end{array}\right)(q-1)^{e-i} .
$$

The two necessary conditions for the existence of a perfect code of order $e$ in $H(n, q)$ mentioned in [3] are.

$$
\sum_{i=0}^{e}\left(\begin{array}{l}
n \\
i
\end{array}\right)(q-1)^{i}=q^{k}
$$

for some integer $k$ (cf. [7]) and Theorem 7 of [3] which states

$$
\left\{\begin{array}{l}
\Psi_{e} \text { has e distinct zeros } x_{1}<x_{2}<\ldots<x_{e} \text { which are integers } \\
\text { in }[1, n] .
\end{array}\right.
$$

The following properties of $\Psi_{e}$ and its zeros are easily obtained by substitution or by calculating suitable coefficients of $\Psi_{e}$.

$$
\begin{aligned}
& \Psi_{e}(0)=\sum_{i=0}^{e}\left(\begin{array}{l}
n \\
i
\end{array}\right)(q-1)^{i}, \\
& \Psi_{e}(1)=\left(\begin{array}{c}
n-1 \\
e
\end{array}\right)(q-1)^{e},
\end{aligned}
$$




$$
\sum_{i=1}^{e} x_{i}=\frac{e(n-e)(q-1)}{q}+\frac{e(e+1)}{2}
$$

$$
\sum_{i=1}^{e} x_{i} \leq \frac{n e(q-1)}{q} \quad(\text { for } q>2), q=3, e \geqslant 3
$$

$$
\prod_{i=1}^{e} x_{i}=e ! q^{-e_{\Psi}} e^{(0)}
$$

$$
\prod_{i=1}^{e}\left(x_{i}-1\right)=e ! q^{-e_{\Psi}} \Psi^{(1)}
$$

For integral values of $\mathrm{x}$ between 1 and $\mathrm{n}$ the terms of the sum in (2.2) alternate in sign. Since the terms decrease in absolute value, with increasing $i$, for $x<\frac{(n-e+1)(q-1)+e}{q-1+e}$ we have (if $\Psi_{e}$ has integral zeros):

$$
x_{1} \geq \frac{(n-e+1)(q-1)+e}{q-1+e} \text {. }
$$

It was suggested by D.H. SMITH that the following lemma could prove to be useful in non-existence proofs of perfect codes.

IEMMA 1. If a non-trivial perfect code of order e in $\mathrm{H}(\mathrm{n}, \mathrm{q})$ exists then (i) $\mathrm{n} \geq \frac{1}{2} \mathrm{e}^{2}+\frac{5}{2} \mathrm{e}+1$ if $\mathrm{q}>2$,

(ii) $n \geq e^{2}+4 e+2$ if $q=2$.

PROOF. Let $Y$ be a non-trivial perfect code of order $e$ and let $\underline{a}=\left(a_{0}, a_{1}, \ldots, a_{n}\right)$ be the distance distribution of $Y$. Then we have

$$
\begin{aligned}
& a_{2 e+1}=e ! n !(q-1)^{e+1}\{(n-e-1) !(2 e+1) !\}^{-1}, \\
& a_{2 e+2}=\frac{1}{2} a_{2 e+1}\left\{\frac{q-1}{e+1}\left(n-e^{2}-3 e-1\right)+e\right\}
\end{aligned}
$$

and

$$
a_{2 e+3}=a_{2 e+1}(n-2 e-1)\left(n-e^{2}-4 e-2\right)\{(2 e+2)(2 e+3)\}^{-1} \text { if } q=2 \text {. }
$$

Then (i) and (ii) follow from the observation that $a_{2 e+2} \geq 0$ and $a_{2 e+3} \geq 0$. $\square$ We need one more concept which will play an important role in the nonexistence proof. We define for $n \in \mathbb{N}$ 


$$
a_{p}(n):=\max \{m \in \mathbb{N}|m| n, p+m\},
$$

i.e. $a_{p}(n)$ is the largest divisor of $n$ which is not divisible by $p$. We call $n_{1}$ and $n_{2}$ p-equivatent if $a_{p}\left(n_{1}\right)=a_{p}\left(n_{2}\right)$.

since one can explicitly determine the zeros of $\Psi_{2}$ it is easy to show that the ternary Golay code is the only non-trivial perfect code of order 2 (cf. [7]). In the following theorem we therefore take $e>2$.

THEOREM 1. If $q=p^{r}, e>2$ then there is no perfect code $\mathrm{y}$ of order $\mathrm{e}$, with $|\mathrm{y}|>2$, in $\mathrm{II}(\mathrm{n}, \mathrm{q})$.

PROOF. Assume $Y$ is a perfect code of ordex $e<(n-1) / 2$ in $H(n, q)$. For the zeros of $\Psi_{e}$ we find from $(2.3),(2.5)$ and $(2.9)$

$$
\prod_{i=1}^{e} x_{i}=e ! q^{k-e}
$$

and hence

$$
a_{p}\left(x_{1}\right) a_{p}\left(x_{2}\right) \ldots a_{p}\left(x_{e}\right)=a_{p}(e !) \leq e ! .
$$

It follows that there are zeros $x_{i}, x_{j}$ which are p-equivalent or $\left\{a_{p}\left(x_{1}\right), a_{p}\left(x_{2}\right), \ldots, a_{p}\left(x_{e}\right)\right\}=\{1,2, \ldots, e\}$, i.e. $p>e \geq 3$. In the latter case there is a zero $x_{i}=p^{\alpha}$ and a zero $x_{j}=2 p^{\beta}$ and then either $x_{i} \geq 2 x_{j}$ or $x_{j} \geq 2 x_{i}$. Hence we always have

$$
\text { (2.14) } 2 x_{1} \leq x_{e} \text { and hence } x_{1} x_{e} \leq \frac{2}{9}\left(x_{1}+x_{e}\right)^{2} \text {. }
$$

Now by $(2.5),(2,8),(2.14)$ and the arithmetic-geometric mean inequality we find

$$
\begin{aligned}
&(q-1)^{e} q^{-e} n(n-1) \ldots(n-e+1)<e ! q^{-e_{\psi}}(0)=\prod_{i=1}^{e} x_{i} \leq \\
& \leq \frac{8}{9}\left(\frac{x_{1}+x_{e}}{2}\right)^{2}\left(\frac{x_{2}+x_{3}+\ldots+x_{e-1}}{e-2}\right)^{e-2} \leq \\
& \leq \frac{8}{9}\left(\frac{x_{1}+\ldots+x_{e}}{e}\right)^{e} \leq \frac{8}{9}(q-1)_{q}^{e}-e_{n}^{e} \\
&\text { (for } q>2) .
\end{aligned}
$$

If $q=2$ the final expression is $\frac{8}{9}\left(\frac{n+1}{2}\right)^{e}$. 
Now let $q>2$. From $(2.6)$ and $(2.10)$ we find $q^{e} \mid(n-1) \ldots(n-e)$ and therefore

$$
n>p^{r e-[e / p]-\left[e / p^{2}\right]-\ldots>p^{e\left(r-\frac{1}{p-1}\right)} \geq q^{\frac{1}{2} e} .}
$$

By (2.15) we have

$$
1-\frac{e(e-1)}{2 n}<\frac{n(n-1) \cdots(n-e+1)}{n^{e}} \leq \frac{8}{9}
$$

i.e.

$$
n<\frac{9}{2} e(e-1)
$$

and hence (2.16) implies

$$
3^{\frac{1}{2} e} \leq q^{\frac{1}{2} e}<\frac{9}{2} e(e-1), \text { i.e. } e \leq 11 \text {. }
$$

It then follows that $\mathrm{q} \leq 8$ and $\mathrm{n} \leq 495$. In 1967 (cf. [9]) a computer search had found all solutions of (2.5) for $n \leq 1000, q \leq 100$, $e \leq 1000$. This yielded no new codes. Hence for $q>2$ the proof is finished.

For the case $q=2(n \neq 2 e+1)$ we do not have an inequality of type (2.16). We now have to use lemma 1 to get a lower bound on $n$ and generalize the method used above to obtain an upper bound. Starting from (2.14) one shows by induction that

$$
\prod_{i=1}^{s} \xi_{i} \leq\left(\frac{8}{9}\right)^{s-1}\left(\frac{1}{s} \sum_{i=1}^{s} \xi_{i}\right]^{s}
$$

if $\xi_{1}, \xi_{2}, \ldots, \xi_{s}$ are 2 -equivalent. Then in the same way as above the arithmetic-geometric mean inequality yields

$$
\prod_{i=1}^{e} x_{i} \leq\left(\frac{8}{9}\right)^{e-m}\left[\frac{x_{1}+\ldots+x_{e}}{e}\right)^{e}
$$

if $x_{1}, x_{2}, \ldots, x_{e}$ are divided over $m$ equivalence classes under 2-equivalence. This means that if we can prove that $m \leq e-6$ then the analogue of (2.15) extended by (2.17) gives us

$$
n(n-1) \ldots(n-e+1)<\left[\frac{8}{9}\right]^{6}(n+1)^{e},
$$

i.e. $n<e^{2}+e$, which contradicts inequality (ii) of lenma 1 .

In the proof of the remaining step we let $p(x)$ denote the product of the odd integers $\leq \mathrm{x}$. Then 


$$
\begin{aligned}
a_{2}\left(x_{1} x_{2} \ldots x_{e}\right) & =a_{2}(e !) \leq p(e)\left[\frac{e}{2}\right]: 2^{-\left[\frac{e}{4}\right]}< \\
& <p(e) e^{\left[\frac{e}{2}\right]-5} \quad(\text { for } e \geq 16) .
\end{aligned}
$$

Fur thermore,

$$
p(e) \leq p(2 m) e^{\left[\frac{e+1}{2}\right]-m}
$$

and

$$
a_{2}\left(x_{1} x_{2} \cdots x_{e}\right) \geq 1 \cdot 3 \cdot 5 \cdots(2 m-1)=p(2 m)
$$

Combining these inequalities we find

$$
1<e^{\left[\frac{e}{2}\right]+\left[\frac{e+1}{2}\right]-m-5},
$$

i.e. $m \leq e-6$ (for $e \geq 16$ ). Hence the proof is complete for $e \geq 16$.

This leaves $e \leq 15$ and then by $(2.15)$ n $<1000$ and we again refer to the computer search.

Admittedly the case $q=2$ is still rather messy. However, it seems likely that further simplifications of the proof are possible. We advise the reader to study the proof given here carefully in order to appreciate the great difficulties that arise when one tries to generalize to values of $\mathrm{q}$ which are not prime powers. In the next section we will see that even the case $e=2$, where one can explicitly determine the zeros of $\Psi e^{\text {' is }}$ difficult.

3. HAMMING SCHEMES $H(n, q)$ WITH $q$ NOT A PRIME POWER

If $q$ is not a prime power the sphere packing condition no longer has the form $(2.3)$ which is replaced by

$$
\sum_{i=0}^{e}\left(l_{i}^{n}\right)(q-1)^{i} \mid q^{n} .
$$

The other necessary condition for the existence of a perfect code still has the form (2.4). 
Condition (3.1) is satisfied for $e=1$ and $n=q+1$. It has been shown that a perfect code of order 1 in $H(7,6)$ does not exist. As far as we know this is the only case where non-existence has been proved (for $q$ not a prime power). Attempts to generalize the non-existence proofs for e $\geq 2$ have failed up to now because (3.1) is so much weaker than (2.3) that as a consequence (2.13) is replaced by a weaker statement. But even if (2.13) remained true the idea of splitting the zeros of $\Psi_{e}$ into equivalence classes, which was the essential step in section 2 , cannot be generalized.

As a small step on the road to complete understanding of perfect codes we shall completely treat the case $q=10, e=2$ since the alphabet of 10 symbols is of practical interest and this case illustrates how some of the ideas of section 2 can still be used.

We assume that a perfect code of order $e$ in $H(n, q)$ exists. From now on we take $q=10$ but continue to use the symbol $q$ in view of application to other examples. We shall keep e arbitrary as long as possible and then specialize to $e=2$. The sphere packing bound now reads

$$
\sum_{i=0}^{e}\left(\begin{array}{l}
n \\
i
\end{array}\right)(q-1)^{i}=q^{k} p^{\alpha}
$$

where $p=2$ or $p=5$. We define $\bar{p}$ by $q=p \bar{p}$. Since $\sum_{i=0}^{n}\left(\begin{array}{l}n \\ i\end{array}\right)(q-1)^{i}=q^{n}$ we find by subtraction that

$$
q^{k} p^{\alpha}\left(q^{n-k-\alpha} \bar{p}^{\alpha}-1\right) \equiv 0\left(\bmod (q-1)^{e}\right)^{q !} \text {. }
$$

For $e \geq 1$ this implies that $\alpha \equiv 0(\bmod 6)$. For the zeros of $\psi_{e}$ we again have (2.8) and (2.11). Instead of (2.y) we have

$$
\prod_{i=1}^{e} x_{i}=e ! q^{k-e} p^{\alpha} \text {. }
$$

Furthermore we find from (2.6) and (2.10) in precisely the same way as (2.16) the inequality

$$
n>5^{\frac{3}{4} e}
$$

(from the fact that $5^{e}$ divides $(n-1) \ldots(n-e)$ ).

Next we remark that the argument of (2.15) still holds if $2 x_{1} \leq x_{e}$. This would again yield the inequality $n<\frac{9}{2} e(e-1)$, which contradicts (3.4). Hence we now have 
(3.5) $2 x_{1}>x_{e}$

THEOREM 2. There is no perfect code of order 2 in $\mathrm{H}(\mathrm{n}, 10)$ for $\mathrm{n}>2$.

ProOF. Assume on the contrary that such a code exists. By the sphere packing bound we have

$$
(18 n-7)^{2}-41=8 q^{k} p^{\alpha}
$$

Since $29^{2}-41=8 q^{2}$ we find by subtraction

$$
36(9 n+11)(n-2)=8\left(q^{k} p^{\alpha}-q^{2}\right) .
$$

Lloyd's theorem states that the zeros $x_{1}, x_{2}$ of

$$
x^{2}-\left\{\frac{9}{5}(n-2)+3\right\} x+2 q^{k-2} p^{\alpha}
$$

are integers between 1 and $n$.

We can already draw the following conclusions:

$$
\mathrm{n} \equiv 2(\bmod 5),
$$

$$
k \geq 2 \text {, }
$$

the latter because $x_{1} x_{2}$ is an integer only if $k \geq 2$ or $k=1$ and $p=5$ but in that case $x_{1} x_{2}=5^{\alpha-1}$ which contradicts $(3.5)$. From (3.9), (3.10) and $(3.7)$ we find that $n \equiv 2(\bmod 25)$ and using this we see from $(3.8)$ that $x_{1}+x_{2} \not \equiv 0(\bmod 5)$. It follows that one of the zeros is a power of 2 . Let

$$
\mathrm{x}_{1}=2^{\nu}, \mathrm{x}_{2}=2^{\mu} 5^{\sigma}
$$

(where we no longer require $x_{1}$ to be the smaller of the two zeros). We consider the equation (3.7) mod 32. The right-hand side is 0 and therefore we have two possibilities to considex, namely $\mathrm{n} \equiv 2(\bmod 8)$ and $\mathrm{n} \equiv 5$ $(\bmod 8)$.

Case $i: n \equiv 2(\bmod 8)$. In (3.8) one of the zeros is odd and since $x_{1} \neq 1$ we must have $\mu=0$. Furthemore, $x_{1}+x_{2}$ is divisible by 3 which implies that $v+\sigma$ is odd. We now return to the sphere packing bound (3.6) with the knowledge that

$$
\begin{aligned}
& \frac{9}{5}(n-2)+3=2^{\nu}+5^{\sigma} \quad(\nu+\sigma \text { odd }) \text {. } \\
& \lambda \neq 1,2 \text {. }
\end{aligned}
$$


Substitution yields

$$
\left(5 \cdot 2^{\nu+1}+2 \cdot 5^{\sigma+1}-1\right)^{2}-41=8 \cdot 10^{k} \cdot p^{\alpha}
$$

i.e.

$$
2^{2} \cdot 5^{2 \sigma+2}-2^{2} \cdot 5^{\sigma+1} \equiv 40(\bmod 32)
$$

which is a contradiction.

Case ii : $\mathrm{n} \equiv 5(\bmod 8)$. We now have $\mathrm{x}_{1}+\mathrm{x}_{2} \equiv 2(\bmod 8)$ and since (2.11) implies $x_{1} \neq 2$ we must have $x_{2}=2 \cdot 5^{\sigma}$. Again we substitute in (3.6). We find

$$
-5 \cdot 2^{v+2} \equiv 40(\bmod 25), \text { i.e. } v \equiv 3(\bmod 4)
$$

and

$$
2^{4} \cdot 5^{2 \sigma+2}-2^{3} \cdot 5^{\sigma+1} \equiv 40(\bmod 64) \text { unless } k=2 \text { and } p=5 \text {. }
$$

$x_{1}=1$ wit If $k=2$ and $p=5$ then $x_{1}=16$ and hence by $(2.11) n \leq 20$ $3.8 \Rightarrow$ contradicting $n \equiv 2(\bmod 25)$. So we must have $\sigma$ even. Then contradictie

$$
x_{1}+x_{2}=2^{\nu}+2 \cdot 5^{\sigma} \equiv 1(\bmod 3)
$$

which contradicts $(3.8)$.

This completes the proof.

of course this is an isolated example of a non-existence proof. In order to generalize this, e.g. to all $q$ which are twice a prime, more ideas are necessary. We hope that some of the ideas used above will prove fruitful in future research on perfect codes.

\section{JOHNSON SCHEMES $J(n, v)$}

Let $\mathrm{n}=2 \mathrm{e}+1$ and $\mathrm{v}=2 \mathrm{n}$. We consider the two word code $Y:=\{(1,1, \ldots, 1,0,0, \ldots, 0),(0,0, \ldots, 0,1,1, \ldots, 1)\}$ which we can interpret as an analogue of the repetition code in a Hamming scheme. Clearly every element of $J(n, v)$ has distance $\leq e$ to exactly one of the elements of $Y$, i.e. $Y$ is a perfect code of order e. These examples and the perfect codes with $|\mathrm{Y}|=1$ and $e=v$ we again consider trivial. No example of a nontrivial perfect code in $J(n, v)$ is known. In a search for such codes one 
quickly sees that it is again the sphere packing bound which is difficult to exploit. We shall briefly illustrate the case $e=2$. We then have the two necessary conditions for the existence of a perfect code of order 2 in $J(n, v)$ :

$$
\begin{aligned}
\left\{1+\left(\begin{array}{c}
n \\
1
\end{array}\right)\left(\begin{array}{c}
v-n \\
1
\end{array}\right)+\left(\begin{array}{l}
n \\
2
\end{array}\right)\left(\begin{array}{c}
v-n \\
2
\end{array}\right)\right\} \mid\left(\begin{array}{l}
v \\
n
\end{array}\right) & \\
4 \Psi_{2}(x) & =x^{2}+\left\{2 n^{2}-2 v n+v-6\right\} x+ \\
& +\left\{n^{4}-2 v n^{3}+\left(v^{2}+v-5\right) n^{2}+\left(-v^{2}+5 v\right) n+4\right\}
\end{aligned}
$$

has two zeros which are both integers of the form $i(v+1-i)$ with $0 \leq i \leq n$. So far the only solutions to these two conditions which we have been able to find correspond to trivial codes.

\section{OTHER METRIC SCHEMES; GRAPHS}

Let $\mathrm{x}$ be the set of rowvectors with 7 coordinates, three of which are 1 and the others 0 . For $\underline{x}, \underline{y} \in \mathrm{X}$ we define

$$
\begin{aligned}
& \rho(\underline{x}, \underline{y})=1 \text { if }(\underline{x}, \underline{y})=0, \\
& \rho(\underline{x}, \underline{y})=2 \text { if }(\underline{x}, \underline{y})=2, \\
& \rho(\underline{x}, \underline{y})=3 \text { if }(\underline{x}, \underline{y})=1,
\end{aligned}
$$

where $(\underline{x}, \underline{y})$ is the inner product over $\mathbb{R}$. It is easy to check that this is a distance function for $x$. With each vector in $x$ we associate a vertex $v(\underline{x})$ of a graph $G$ and we join the vertices $v(\underline{x})$ and $v(\underline{y})$ by an edge iff $\rho(\underline{x}, \underline{y})=1$. It turns out that $\rho(\underline{x}, \underline{y})$ is the distance of $v(\underline{x})$ and $v(\underline{y})$ in the graph $G$. It is straightforward to check that the distance $\rho$ defines a metric scheme in the sense of Remark (ii) of $[3,52]$.

$G$ is a perfectly regular graph with valency $v_{1}=4$. Let $Y$ be the set of 7 rowvectors of the incidence matrix of $P G(2,2)$. If $\underline{x}$ and $\underline{y}$ are two distinct rows of $\mathrm{y}$ then clearly $\rho(\underline{\mathrm{x}}, \underline{\mathrm{y}})=3$. Since $|\mathrm{x}|=35$ we have

$$
\left(1+v_{1}\right)|y|=|x| \text {, }
$$

i.e. equality in the sphere packing bound $[3, \S 4.1$, formula (3)]. Hence $Y$ is a perfect code of order 1 in the metric scheme. This is the first example given by BIGGS [1] in his paper on perfect codes in distance- 
transitive graphs. In [2] a number of other examples is given, all with $e=1$. We have illustrated the example here in the setting of [3]. In both points of view it is clear that proving that $Y$ is a perfect code is easy compared to showing that we have a metric scheme to start with. In the terminology of graph theory the difficult problem is to show that $G$ is a distance-transitive graph and not to find the perfect code. Theorems of the type we discussed for the Haming schemes were possible because we had an infinite class of schemes in which we could search for perfect codes. It does not seem likely that this will be the case for distance-transitive graphs. So even though we still have Lloyd's theorem as a tool we do not know where to use it. It would be extremely interesting if a perfect code of order $e>1$ would be found in a scheme of the type considered here. of course the Golay code is such a code and there is a code derived from the Golay code which is also of order 3 (O. HEDEN, private communication) but this code is essentially the same as the Golay code. An example not corresponding to a Hamming scheme is not known.

\section{NEARLY PERFECT CODES}

In this section we discuss a class of completely regular codes namely the nearly perfect codes introduced by GOETHALS \& SNOVER [5].

JoHNSON [6] proved the following extension of the sphere packing bound (see also section 7):

LEMMA 2. If $\mathrm{Y}$ is a code with minimum distance $\mathrm{d}=2 \mathrm{e}+1$ in $\mathrm{x}:=\mathrm{H}(\mathrm{n}, 2)$ then

$$
|Y|\left\{\sum_{i=0}^{e}\left(\begin{array}{l}
n \\
i
\end{array}\right)+\frac{1}{[n /(e+1)]}\left(\begin{array}{l}
n \\
e
\end{array}\right)\left(\frac{n-e}{e+1}-\left[\frac{n-e}{e+1}\right]\right)\right\} \leq 2^{n}=|x| .
$$

If e+1 divides $n+1$ then this reduces to the sphere packing bound. It is well known that $(e+1) \mid(n+1)$ is a necessary condition for the existence of a perfect code in $H(n, 2)$. The code $Y$ is called nearly perfect if equality holds in (6.1). From the proof of (6.1) it immediately follows that if $Y$ is a nearly perfect code then for every $\mathrm{X} \in \mathrm{X}$ with $\rho(\underline{x}, \mathrm{Y})>e$ there are exactly $[\mathrm{n} /(\mathrm{e}+1)]$ points $\underline{\mathrm{y}} \in \mathrm{Y}$ with $\rho(\underline{x}, \underline{y})=, e+1$. Furthermore, it follows that if $\rho(\underline{x}, \underline{y})=$ e for some $\underline{y} \in Y$, then there are exactly $[(n-e) /(e+1)]$ points $\underline{z} \in Y$ with $\rho(\underline{x}, \underline{z})=e+1$. In fact, such a code $Y$ is completely regular. The distance distribution of $\mathrm{Y}$ is determined in [5]. 
The following theorem is an example of the theorems given in [5] showing the importance of nearly perfect codes in the theory of designs. We shall interpret a point of $\mathrm{Y}$ as the incidence vector of a subset of a set $\mathrm{s}$ of $\mathrm{n}$ points.

THEOREM 3. If $\mathrm{Y}$ is a nearly perfect code with minimum distance $\mathrm{d}=2 \mathrm{e}+1$ in $\mathrm{X}=\mathrm{H}(\mathrm{n}, 2)$ and $\underline{\mathrm{g}} \in \mathrm{Y}$ then the words of weight $\mathrm{d}$ in $\mathrm{Y}$ form an e-design with $\lambda=[(n-e) /(e+1)]$.

PROOF. AnY e-subset $D$ of $S$ corresponds to a point $\underline{x} \in \mathrm{X}$ with weight e, i.e. distance $e$ to $\underline{0}$. We mentioned above that $\underline{x}$ then has distance e+1 to exactly $\lambda$ points of $Y$ each of which therefore has weight $2 e+1$. Hence $D$ is a subset of exactly $\lambda$ sets corresponding to code words of weight $2 e+1$.

It is also shown in [5] that such designs can be extended to (e+1)designs.

The non-linear codes known as the Preparata codes (cf. [10]) have $\mathrm{n}=4^{\mathrm{m}}-1,|\mathrm{y}|=2^{\mathrm{n}-\mathrm{r}}$ where $\mathrm{r}=4 \mathrm{~m}-1 \quad(\mathrm{~m} \geq 2)$, and $\mathrm{d}=5$. By substitution we see that these codes satisfy (6.1) with equality, i.e. they are nearly perfect. We thus obtain an infinite class of 3-designs.

of course the question now rises whether nearly perfect codes with $e>2$ can be found. (From now on we exclude perfect codes.) The definition alone is enough to show that the answer is negative for $e=3$ and $e=4$. GOETHALS \& SNOVER mention this in their paper without giving the proof. We present their proof here.

THEOREM 4. Except for the known perfect codes there are no nearly perfect codes with minimum distance 7 or 9.

\section{PROOF.}

(i) Suppose $n \not z 3(\bmod 4)$. Let $n+1 \equiv s(\bmod 4)$ where $s=1,2$ or 3 . Then substituting this and $e=3$ in (6.1) yields

$$
|y|\left\{1+n+\left(\begin{array}{l}
n \\
2
\end{array}\right)+\frac{n+1}{n+1-s}\left(\begin{array}{l}
n \\
3
\end{array}\right)\right\}=2^{n} \text {. }
$$

This can be written as

$$
|y|\left\{6+(n+1)\left(n^{2}+(s-1) n+(s-2)(s-3)\right)\right\}=3 \cdot 2^{n+1}
$$

If $s=1$, i.e. $n \equiv 0(\bmod 4)$, we find from (6.3) 


$$
|y|(n+2)\left(n^{2}-n+4\right)=3 \cdot 2^{n+1}
$$

and hence $(n+2) \mid 6$ which gives us $n=4$, which does not correspond to a nearly perfect code. If $s=2$ or 3 we obtain from (6.3)

$$
|y|(n+1) n(n+s-1)=6\left\{2^{n}-|y|\right\}
$$

where $|\mathrm{y}|=2^{\mathrm{k}}$ or $3 \cdot 2^{\mathrm{k}}(\mathrm{k}<\mathrm{n})$. This implies that

$$
(n+1) n(n+s-1)=6\left(2^{n-k}-1\right) \text { or } 2\left(2^{n-k}-3\right) \text {. }
$$

Here the left-hand side is $\equiv 0(\bmod 4)$ and the right-hand side is $\equiv 2(\bmod 4)$, a contradiction.

(ii) We now consider the case $e=4$. Let $n+1 \equiv s(\bmod 5)$, where $s=1,2,3$ or 4. Again we substitute in (6.1) and replace $n+1$ by $m$. We find

$$
3 \cdot 2^{n+3} /|\mathrm{y}|= \begin{cases}\mathrm{m}\left(\mathrm{m}^{3}-5 \mathrm{~m}^{2}+14 m+8\right) & \text { if } \mathrm{s}=1, \\ \mathrm{~m}\left(\mathrm{~m}^{3}-4 \mathrm{~m}^{2}+7 m+20\right) & \text { if } \mathrm{s}=2, \\ \mathrm{~m}\left(\mathrm{~m}^{3}-3 \mathrm{~m}^{2}+2 m+24\right) & \text { if } \mathrm{s}=3, \\ \mathrm{~m}\left(\mathrm{~m}^{3}-2 \mathrm{~m}^{2}-\mathrm{m}+26\right) & \text { if } \mathrm{s}=4 .\end{cases}
$$

Clearly 16 t $m$ and therefore $m \mid 24$ which leaves only a finite number of cases which are all easily ruled out.

Of course the first really interesting case is e $=5$ since it could lead to a 6-design. In this case we were not able to do anything with (6.1) alone. However, GOETHALS \& SNOVER also proved that there is an analogue of Lloyd's theorem for nearly perfect codes. We quote the theorem.

THEOREM 5. Let there exist a nearly perfect code in $\mathrm{H}(\mathrm{n}, 2)$ with minimum distance $2 \mathrm{e}+1$ and let $\mathrm{n}+1 \neq 0(\bmod e+1)$. Then the polynomial

$$
Q(x):=\Psi_{e-1}(x)+\frac{1}{[(n+1) /(e+1)]}\left(\Psi_{e+1}(x)-\Psi_{e-1}(x)\right)
$$

(where $\Psi_{\mathrm{e}}(\mathrm{x})$ is the polynomial of $(2,2)$ with $\mathrm{q}=2$ ) has e+1 distinct integral zeros between 1 and $\mathrm{n}$.

As was to be expected the case $e=5$ does not yield any solutions either. 
THEOREM 6. There is no nearly perfect code with minimum distance 11 in $\mathrm{H}(\mathrm{n}, 2)$ for $\mathrm{n}>11$.

PROOF. Assume that $Y$ is such a nearly perfect code. We write $n=6 \mathrm{v}+l$ where $\ell=0,1,2,3$ or 4 (since $\ell=5$ is excluded by the theorem on perfect codes). By substitution in (6.1), taking $e=5$, we see that $|y|$ is either a power of 2 or 5 times a power of 2 . Hence we have

$$
\sum_{i=0}^{5}\left(\begin{array}{l}
n \\
i
\end{array}\right)+\frac{1}{[n / 6]}\left(\begin{array}{l}
n \\
5
\end{array}\right)\left\{\frac{n-5}{6}-\left[\frac{n-5}{6}\right]\right\}=2^{r} / a
$$

where $a=1$ or 5 . As in the case of perfect codes the left-hand side of $(6.5)$ is $Q(0)$. By substitution we see that $Q(1)>0$.

In the same way as (2.7) and (2.9) we find the surn and product of the zeros of $Q$ :

$$
\sum_{i=1}^{6} x_{i}=3(n+1)
$$

$$
\prod_{i=1}^{6} x_{i}=\left[\frac{n+1}{6}\right] 6: 2^{r-6} / a
$$

We observe that $Q(n+1-x)=Q(x)$, i.e.

$$
x_{7-i}=n+1-x_{i} \quad(i=1,2,3) \text {. }
$$

We now introduce the variable $z:=(2 x-n-1)^{2}$. On substitution in (6.4) we then find that

$$
\begin{aligned}
Q^{*}(z): & z^{3}+5(-2 n+5-l) z^{2}+\left\{15 n^{2}+10(3 \ell-5) n-(70 \ell-19)\right\} z+ \\
& -15(\ell+1)(n-1)(n-3)
\end{aligned}
$$

has three integral zeros $z_{i}:=\left(2 x_{i}-n-1\right)^{2}, i=1,2,3$. Again a simple computation shows that $Q^{*}(0)<0$ and $Q^{*}(8)>0$. Hence by theorem 5 either $n$ is even and $Q^{*}(1)=0$ or $\mathrm{n}$ is odd and $Q^{*}(4)=0$. However $Q^{*}(4)<0$ for $\ell>0$ and $Q^{*}(1)=-15 \ell(n-2)(n-4)=0$ only if $l=0$. Hence we now know that $n=6 \mathrm{v}$ and that $x_{3}=3 v$ and $x_{4}=3 v+1$. Furthermore, $z_{1}$ and $z_{2}$ satisfy

$$
z^{2}+(26-10 n) z+15(n-1)(n-3)=0,
$$

i.e.

$$
z_{1,2}=5 n-13 \pm\left(10 n^{2}-70 n+124\right)^{\frac{1}{2}}=5 n-13 \pm x,
$$


where

$$
5(2 n-7)^{2}+3=2 x^{2}
$$

Substituting $x_{3}=3 v$ and $x_{4}=3 v+1$ in $(6.7)$ we find

$$
(3 v+1) x_{1} x_{2} x_{5} x_{6}=3 \cdot 5 \cdot 2^{r-2} / a
$$

so either $3 \mathrm{v}+1=2^{\sigma}$ or $3 \mathrm{v}+1=5 \cdot 2^{\sigma}$, i.e. $\mathrm{n}=2\left(2^{\sigma}-1\right)$ or $\mathrm{n}=2\left(5 \cdot 2^{\sigma}-1\right)$. First substitute $\mathrm{n}=2\left(2^{\sigma}-1\right)$ in $(6.9)$. This yields

$$
16\left(5 \cdot 2^{2 \sigma-1}-55 \cdot 2^{\sigma-2}+19\right)=x^{2} \text {. }
$$

The expression in brackets is $\equiv 3(\bmod 4)$ if $\sigma \geq 4$. Hence only $n=14$ is a possibility, but this does not yield a solution. Substitution of $n=2\left(5 \cdot 2^{\sigma}-1\right)$ yields a contradiction in exactly the same way.

\section{UNIFORMLY PACKED CODES}

The uniformly packed codes were introduced by SEMAKOV, ZINOV'EV \& ZAITSEV [11]. Once again the codes are in $\mathrm{H}(\mathrm{n}, 2)$. The definition generalizes the idea of perfect and nearly perfect codes (in fact these are uniformly packed).

Let $Y$ be a binary code of length $n$ and minimum distance $a$. Let $e:=[(d-1) / 2]$. The set of all words of length $n$ is again denoted by $x$. We define

$$
\underline{Y}_{e}:=\{\underline{x} \in X \mid \rho(\underline{x}, y) \geq e\},
$$

and

$$
\forall \underline{z} \in Y_{e},\left[r(\underline{z}):=\left|Y \cap S_{e+1}(\underline{z})\right|\right],
$$

i.e. $r(\underline{z})$ is the number of points $\underline{y} \in Y$ with $e \leq \rho(\underline{y}, z) \leq e+1$. clearly we have

$$
\forall \underline{z} \in Y_{e},[r(\underline{z}) \leq[(n+1) /(e+1)] .
$$

Since

$$
\sum_{\underline{z} \in Y} r(\underline{z})=\sum_{\underline{y} \in Y}\left|S_{e+1}(\underline{y}) \backslash s_{e-1}(\underline{y})\right|=|Y|\left(\left(\begin{array}{l}
n \\
e
\end{array}\right)+\left(\begin{array}{c}
n \\
e+1
\end{array}\right)\right)
$$


and

$$
\left|y_{e}\right|=2^{n}-|y| \sum_{i=0}^{e-1}\left(\begin{array}{l}
n \\
i
\end{array}\right)
$$

we find that the average value $r$ of $r(z)$ is

$$
r=\frac{|Y|\left(\begin{array}{l}
n+1 \\
e+1
\end{array}\right)}{2^{n}-|y| \sum_{i=0}^{e-1}\left(\begin{array}{l}
n \\
i
\end{array}\right)} .
$$

Observe that (7.3) and (7.4) together yield a proof of the Johnson bound (6.1).

If $\forall \underline{z} \in Y_{e},[r(z)=r]$ the code $Y$ is called uniformly packed. clearly a uniformly packed code with $r=[(n+1) /(e+1)]$ is nearly perfect (and of course perfect if $r=(n+1) /(e+1)$ and also if $r=1)$. From now on we only consider uniformly packed codes with $1<r<[(n+1) /(e+1)]$. In $[11]$ the distance distribution of a uniformly packed code is determined and it turns out that these codes are also completely regular. Furthermore, theorem 3 also generalizes. In fact, in the extended code of a uniformly packed code the words of a given weight $\mathrm{w}$ form an (e+1)-design. So once again the search starts:

We use the following notation

$$
r=(n-s) /(e+1) \text {, where } s \geq e .
$$

We restrict the search to $e=1, s \leq 15$ and $e=2, s \leq 5$.

From (7.4) we find

$$
2^{n}(n-s)=|y| \quad\left(n^{2}+2 n-s\right)
$$

Since

$$
n^{2}+2 n-s>(n-s)(n+s+2)
$$

and

$$
n+s+2 \geq 2 s+6 \geq 8 \text {, }
$$

we see that $16 \mid\left(n^{2}+2 n-s\right)$, i.e. $s \equiv 0,3,8$ or $15(\bmod 16)$.

(a) $e=1, s=3$. There are two cases to consider. If 3 in we have

$$
(n+3)(n-1)=n^{2}+2 n-3=2^{k},
$$


i.e. $n=5$, contradicting $r>1$. If $n=3 m$ we find

$$
(3 m-1)(m+1)=2^{k} \text {, }
$$

i.e. $\mathrm{m}=1$ or $\mathrm{m}=3$. So we have a possible solution with $\mathrm{n}=9$ and $r=3$. Then $|y|=2^{5}$. There is indeed a uniformly packed code with these parameters.

Let $C:=$ circulant $(0,1,1,0)$. Then the rows of

$$
G:=\left(\begin{array}{c:ccccc} 
& 1 & 1 & 1 & 1 & 1 \\
I_{5} & & & & &
\end{array}\right)
$$

generate a 5-dimensional linear subspace $Y$ of $X$. The rows of $G$ have weight $\geq 3$ (equality occurring) and no two rows of $G$ coincide in the final 4 positions. Hence the non-zero elements of $Y$ have weight $\geq 3$. We know that the maximal value of $r(\underline{z})$ for $z \in Y_{1}$ is at most 5 and the average value of $r(\underline{z})$ is 3 . Suppose there is a $\underline{z} \in Y_{1}$ with $r(\underline{z})=5$. Then without loss of generality we may assume $w(z)=1$ and then the sum of the 4 points of $Y$ at distance 2 from $z$ would have to be $(1,1, \ldots, 1)-\underline{z}$. Since $(1,1, \ldots, 1) \in Y$ this would $i m p l y \underline{z} \in \mathrm{Y}$, a contradiction. If for some $\underline{z}$ we would have $r(z)=4$ then again without loss of generality we may take $w(\underline{z})=1$ or 2 . If $w(\underline{z})=1$ then the sum of the 3 points of $Y$ at distance 2 from $z$ would have weight 7 , again $a$ contradiction. If $w(\underline{z})=2$ then the sum of the 4 points of $y$ at distance 2 from $\underline{z}$ would have weight 8 , once more a contradiction. This proves that $Y$ is uniformly packed. The 2-design formed by the words of weight 4 of the extended code $\vec{Y}$ is the residual of the symmetric $(16,6,2)$-design.

(b) $\mathrm{e}=1, \mathrm{~s}=8$. Now $(7.6)$ reads

$$
2^{n}(n-8)=|y|\left(n^{2}+2 n-8\right)=|y|(n+4)(n-2) .
$$

If $\mathrm{n} \equiv 2(\bmod 3)$ then $\mathrm{n}=9 \mathrm{~m}+8$ and we find

$$
m \cdot 2^{n}=|y|(3 m+4)(3 m+2)
$$

which implies $m=0$. If $n \neq 2(\bmod 3)$ then $n-2=2^{k}, n+4=2^{l}$, i.e. $n=4$, a contradiction.

(c) $e=1, s=15$. In this case we find from $(7.6)$

$$
(n-15) 2^{n}=|y|(n+5)(n-3) \text {. }
$$


As before we distinguish 4 cases depending on the g.c.d. of $\mathrm{n}$ and 15 . In the same way as above we then find two possible parameter sets for uniformly packed codes:

$$
\begin{aligned}
& n=27, r=6,|\dot{y}|=2^{21}, \\
& n=35, r=10,|y|=2^{29} .
\end{aligned}
$$

At present we do not know whether such codes exist.

We turn to the case $e=2$. Then $n \equiv s(\bmod 3)$ and $n \geq s+6$. We find from (7.4) the equation

$$
(n-s) 2^{n+1}=|y|(n+1)\left(n^{2}+n-2 s\right)
$$

and we observe that

$$
(n-s, n+1)=(s+1, n+1),
$$

(d) $\mathrm{e}=2, \mathrm{~s}=2$. We then have from $(7.9),(7.10)$ and $(7.11)$

$$
\begin{aligned}
& n+1=3 \cdot 2^{k}, \\
& n^{2}+n-4=2^{m} .
\end{aligned}
$$

If $\mathrm{k}>2$ then $\mathrm{m}=2$ which is impossible. If $\mathrm{k}=2$ we find $\mathrm{n}=11$ which yields a possible set of parameters: $n=11, r=3,|y|=24$. We now demonstrate a uniformly packed code with these parameters. Consider a Hadamard matrix $\mathrm{H}_{12}$ of order 12. From

$$
A:=\left(\begin{array}{l}
\frac{1}{2}\left(\mathrm{H}_{12}+J\right) \\
\frac{1}{2}\left(-\mathrm{H}_{12}+J\right)
\end{array}\right)
$$

we leave out the first column. The rows of the remaining matrix are the 24 words of the punctured Hadamard code $y$. From the properties of the Hadamard matrix it follows that $Y$ has minimum distance 5. For any $\underline{z} \in Y_{2}$ we know that $r(\underline{z}) \leq 4$ and the average value of $r(\underline{z})$ is 3 . Suppose $r(\underline{z})=4$ for some $\underline{z}$. This implies that after a suitable multiplication of rows and columns by -1 and a permutation of columns there are 4 rows of $\mathrm{H}_{12}$ which have the form 


$$
\begin{array}{llll}
\mathrm{x}_{1}++ & +++ & +++ & +++ \\
\mathrm{x}_{2}-- & --- & +++ & +++ \\
\mathrm{x}_{3}-- & +++ & --- & +++ \\
\mathrm{x}_{4}-- & +++ & +++ & ---
\end{array}
$$

(where in this notation $z$ corresponds to $(x--\quad+++\quad+++\quad+++))$. Taking $x_{1}=+1$ we must have $x_{2}=x_{3}=x_{4}=-1$ and then there is no other row of $12+1$ 's and -1 's which is orthogonal to these 4 rows, a contradiction. Hence there is no $\underline{z}$ with $r(\underline{z})=4$, and therefore the code $Y$ is uniformly packed. If we extend $Y$ we find $A$. The words of weight $\sigma$ are obtained by leaving out the first and thirteenth row of $A$ (if $A$ has standard form). This yields a well-known 3-design.

(e) $e=2, s=3$. From (7.9), (7.10), (7.11) we find $n+1=2^{k}$. Since $\mathrm{n} \equiv 0(\bmod 3) \mathrm{k}$ must be even and $\mathrm{k} \geq 4$. Then

$$
n^{2}+n-2 s=2^{2 k}-2^{k}-6=3 \cdot 2^{m}
$$

i.e. $\mathrm{m}=1$, a contradiction.

(f) $e=2, s=4$ and $e=2, s=5$ are treated in exactly the same way. We omit the details. No possible parameter sets come up.

The equation (7.4) has a number of infinite families of solutions. We mention one below, the others are still being investigated. Without going into details we mention that there is also a generalization of Lloyd's theorem for uniformly packed codes. In some cases the infinite families of solutions of (7.4) also satisfy the conditions of this theorem.

Let $k \geq 2, n=2^{2 k-1}-1, e=2, r=\frac{1}{3}\left(4^{k-1}-1\right), d=n-2(2 k-1)$ and $|Y|=2^{Q}$. Then these numbers satisfy $(7.4)$. For $k=2$ these are the parameters of the repetition code (which is perfect). For $k \geq 2$ the parameters are those of the 2-error-correcting primitive binary BCH-codes of length $n$ and dimension d (cf. [7]). These codes axe indeed uniformly packed and therefore we find from these codes several infinite sequences of 3-designs. We leave the details for a later paper. 
REFERENCES

[1] BIGGS, N.I., Perfect codes in graphs, J. Combinatorial Theory B, 15 (1973) 289-296.

[2] BIGGS, N.L., Perfect codes and distonce-transitive graphs, Proc. of the British Comb. Conference, Aberystwyth 1973, London Math. Soc. Lecture Notes (to appear).

[3] DELSARTE, P., The association schemes of coding theory, in: Combinatorics, M. HALL, JR. \& J.H. VAN LINT (eds.), Mathematical Centre Tracts 55, Amsterdam, 1974, pp. 139-157.

[4] DELSARTE, P. \& J.M. GOETHALS, Unrestricted codes with the Golay parameters are unique, Report R 238, M.B.L.E. Research Iraboratory, Brussels, 1973.

[5] Goethals, J.M. \& S.L. SNOver, Nearly perfect binory codes, Discrete Math., 3 (1972) 65-88.

[6] JoHNSON, S.M., A new upper bound for expor-corpecting codes, IEEE Trans. Information Theory, IT-8 (1962) 203-207.

[7] LINT, J.H. VAN, Coding theory, Lecture Notes in Mathematics 201, Springer-Verlag, Berlin, 1971.

[8] LINT, J.H. VAN, Nonexistence theorems for perfect error correcting codes, in: Computers in algebra and number theory, SIAM-AMS Proceedings IV, Amer. Math. Soc., Providence, R.I., 1971, pp. 89-95.

[9] LINT, J.H. VAN, 1967-1969 Report of the discrete mathematics group, Report 69-WSK-04 of the Technological University, Eindhoven, 1969 .

[10] PREPARATA, F.P., A class of optimum nonlinear double-errop-correcting codes, Information and Control, 13 (1968) 378-400.

[11] SEMAKOV, N.V., V.A. ZINOV'EV \& G.V. ZAITSEV, Uniformly packed codes, Problems of Information Transmission, 7 (1971) 30-39 (translated from Problemy Peredači Informacii, 7 (1971) 38-50). 
[12] SNover, S.L., The uniqueness of the Nordstrom-Robinson and the Golay binary codes, thesis, Michigan State Univ., 1973.

[13] TIETÄVÄINEN, A., On the nonexistence of perfect codes over finite fields, SIAM J. Appl. Math., 24 (1973) 88-96.

[14] TIETAYVAINEN, A., A short proof for the nonexistence of unknown perfect codes, to appear in Ann. Acad. Sci. Fenn.

[15] ZINOV'EV, V.A. \& V.K. IEONT'EV, A theorem on the nonexistence of perfect codes over finite fields, Problemy Peredači Informacii, to appear (in Russian). 